\title{
In vivo platforms for analysis of HIV persistence and eradication
}

\section{J. Victor Garcia}

Division of Infectious Diseases, UNC Center for AIDS Research, University of North Carolina School of Medicine, Chapel Hill, North Carolina, USA.

\begin{abstract}
HIV persistence in patients undergoing antiretroviral therapy is a major impediment to the cure of HIV/AIDS. The molecular and cellular mechanisms underlying HIV persistence in vivo have not been fully elucidated. This lack of basic knowledge has hindered progress in this area. The in vivo analysis of HIV persistence and the implementation of curative strategies would benefit from animal models that accurately recapitulate key aspects of the human condition. This Review summarizes the contribution that humanized mouse models of HIV infection have made to the field of HIV cure research. Even though these models have been shown to be highly informative in many specific areas, their great potential to serve as excellent platforms for discovery in HIV pathogenesis and treatment has yet to be fully developed.
\end{abstract}

\section{HIV persistence}

Since the early days of highly active antiretroviral therapy (HAART), it has been clear that, despite strong and long-lasting suppression of the virus in peripheral blood, HIV can persist in patients indefinitely. Moreover, interruption of treatment leads to a rapid rebound of plasma viremia to levels similar to those existing prior to therapy initiation (1-6). This recrudescence is believed to be the result of the reactivation of latently infected cells present in the peripheral blood and tissues of ART-suppressed patients. Latently infected $\mathrm{T}$ cells in the peripheral blood represent the most extensively studied and best-characterized HIV reservoir in patients. The contribution of other hematopoietic cells to HIV persistence is currently the subject of intense investigation and debate (7-9). However, little information is currently available regarding latently infected cells in tissues.

In the literature, the term "HIV reservoir" has been used to indicate a number of different measures of HIV, such as viral DNA (vDNA), vRNA, viral proteins, and virus outgrowth from T cells stimulated ex vivo. The overall HIV reservoir contributing to persistence in ART-treated individuals might include some low level of actively replicating virus or chronically infected cells. For the purposes of this Review, we will define the latent HIV reservoir (i.e., not expressing functional proviruses) as the absolute number of long-lived, resting, latently infected cells that, upon stimulation in vivo or ex vivo, can produce replication-competent virus capable of reestablishing the infection. We use this strict definition because it most closely describes the target that must ultimately be destroyed to truly rid the body of infection. Therefore, the HIV reservoir is composed of all the latently infected, long-lived, resting, inducible cells present in all tissues and the peripheral blood of an infected individual.

The gold standard for measurement of the HIV reservoir in patients is the quantitative viral outgrowth assay (QVOA) per-

Conflict of interest: The author has declared that no conflict of interest exists. Reference information: / Clin Invest. 2016;126(2):424-431. doi:10.1172/JCI80562. formed on resting peripheral blood cells obtained from fully suppressed patients that are induced ex vivo using maximal stimulation to produce virus (10-14). This peripheral blood reservoir of latently infected cells has been shown to persist for decades and to have an extremely long half-life $(13,15)$. However, it is likely that in humans, long-lived, resting, latently infected cells also exist in tissues (1). Some tissues including the brain have been referred to as sanctuaries for viral persistence, because infected cells located within these tissues may be extremely difficult to eliminate due to a lack of drug penetrance $(16,17)$. Currently, there are ethical and practical issues that prevent the study of the HIV reservoir in human tissues. Similar ethical and practical considerations prevent the evaluation of novel clinical approaches to HIV eradication, because their implementation might pose unnecessary risk to an otherwise relatively healthy group of individuals undergoing safe and effective treatment for HIV infection $(18,19)$. Therefore, detailed investigation of the molecular basis of HIV persistence in vivo, as well as in vivo evaluation of novel approaches to HIV eradication, require the use of animal models that faithfully (or at least adequately) reflect key aspects of the human condition. The two types of models currently available for this type of research are nonhuman primates (NHPs) and humanized mice. This Review will examine the utility of these models to investigate HIV latency, persistence, reactivation, and eradication, with an emphasis on recent work done in humanized mice.

\section{HIV species specificity and tropism}

HIV is a pathogen of humans and is not known to cause disease in any other species. Although HIV is human tropic, it can also replicate in chimpanzees (20-24). HIV cannot infect or replicate in other species of commonly used laboratory animals like mice, rats, rabbits, and macaques (25-29). The species specificity of HIV for humans is multifactorial and not limited to the interactions of viral surface proteins with human cell-surface receptors. For example, coexpression of the cell-surface HIV receptor (human CD4) and either of its coreceptors (CCR5 or 
Table 1. Mouse strains commonly used to make humanized mice to study HIV latency, persistence, and eradication strategies

\begin{tabular}{|c|c|c|}
\hline Strain & Abbreviation & Models for which it has been used \\
\hline Prkdc sclo & SCID & thy/liv implant \\
\hline NOD.CB17-Prkde SCIO $/]^{-}$ & NOD/SCID & BLT \\
\hline NOD.Cg-Prkdc $\left.{ }^{S C I D} \| 2 \operatorname{rg}^{\text {tmiWi }} / S z\right]$ & NSG & BLT, CD34 transplant and ToM \\
\hline $\mathrm{NOD} \cdot$ Rag1 $^{1--} \mathrm{IL} 2 \mathrm{r}^{-1-}$ & NRG & CD34 cell transplant \\
\hline $\mathrm{BALB} / \mathrm{c}-\operatorname{Rag}^{1-1-} \gamma \mathrm{c}^{-/-}$ & DKO & CD34 cell transplant \\
\hline
\end{tabular}

CXCR4) does not render mouse or rat cells competent to support virus replication $(26,29)$. Viral restriction factors, including tripartite motif-containing protein $5 \alpha$ (TRIM $5 \alpha$ ) and apolipoprotein B mRNA-editing enzyme, catalytic polypeptide 3 (APOBEC3), also severely limit the ability of HIV to replicate in other species (30-36). These barriers to HIV replication in multiple species have resulted in a lack of adequate animal models to study basic aspects of HIV replication, pathogenesis, latency, and persistence.

\section{An introduction to humanized mouse models}

In the early 1980s, pioneering work from the laboratory of John E. Dick and others established the repopulation of immunodeficient mice with human hematopoietic stem cells (HSCs) (37-42). Since then, many different types of "humanized mice" have been described, and these models have been reviewed extensively elsewhere (43-46). Virtually all modern humanized mouse models (Figure 1) are produced via transplantation of human $\mathrm{CD}_{34} 4^{+} \mathrm{HSCs}$ and/or human tissues into one of several different strains of immunodeficient mice (46), resulting in systemic or local reconstitution with human hematopoietic cells that, depending on the model, can include human B cells, monocytes and macrophages, DCs, and T cells. When humanized mice are generated via the transplantation of human $\mathrm{CD} 34^{+}$ stem cells, human $\mathrm{T}$ cells are produced in only certain strains. In these specific mouse strains, the human $\mathrm{T}$ cells generated from transplanted $\mathrm{CD}_{3} 4^{+}$cells are produced in the mouse thymus and are presumed to be educated in the context of mouse MHC class I and II (47-49). However, in humanized mouse models in which human liver and thymus tissues are implanted under the kidney capsule to create a functional human thymus (SCIDhu thy/liv mice, T cell-only mice [ToM], and bone marrow/ liver/thymus [BLT] mice), $\mathrm{T}$ cells can develop in the presence of human thymic epithelium, resulting in HLA I and II restriction $(50,51)$. BLT mice differ from SCID-hu thy/liv mice and ToM, in that they receive an autologous human bone marrow transplant after the implantation of human liver and thymus tissues from which $\mathrm{T}$ cell progenitors and other human hematopoietic cells are derived, resulting in systemic reconstitution with virtually all other types of human hematopoietic cells $(52,53)$. Additionally, several new strains of mice have recently been used to generate humanized mice, some of which have been used to study relevant aspects of HIV persistence in vivo. Table 1 describes the different mouse strains used for the work discussed herein.

\section{Reconstitution of immunodeficient mice with CD34+ cells}

As discussed above, John Dick and colleagues demonstrated that immunodeficient mice could be partially reconstituted with human cells after bone marrow transplantation with human $\mathrm{CD} 34^{+}$cells $(37,38,42)$. Early on, relatively low levels of reconstitution were noted when using several different strains of immunodeficient mice. Subsequently, significantly better levels of human reconstitution were observed when NOD/SCID mice were transplanted with human CD $34^{+}$cells $(39,41,42)$. Surprisingly and almost invariably, only human $\mathrm{B}$ and myeloid cells were present in the reconstituted mice. For the most part, all of the mice reconstituted with $\mathrm{CD} 34^{+}$ cells were devoid of human $\mathrm{T}$ cells. Obviously, this represented a significant limitation for the use of these models in HIV research. The lack of T cells in NOD/SCID mice was overcome by implantation of human thymic tissue, as indicated below for BLT mice (53). In addition, three newly introduced immunodeficient mouse strains, Rag1 $1^{-/}$common gamma chain $^{-/}$(DKO), NOD Rag1 ${ }^{-/} \mathrm{com}^{-}$ mon gamma chain ${ }^{-1}(\mathrm{NRG})$, and NSG mice, were able to produce human $T$ cells using the endogenous mouse thymus (47-49). Since then, numerous additional strains of immunodeficient mice have been shown to support endogenous human $\mathrm{T}$ cell production after bone marrow transplantation with human $\mathrm{CD} 34^{+}$cells (54-57). Some of these models have been used to study HIV persistence.

\section{Strengths and limitations of current humanized mouse models}

The humanized mouse models currently available for biomedical research differ in their level of complexity. In all cases, they are capable of replicating both HIV-1 and -2. Viral replication takes place in human cells present in both the peripheral blood and tissues. The human cells present in these animals have been shown to induce both innate and adaptive immune responses $(46,53,58-61)$. In addition, HIV infection in these models responds to the same drugs used in humans (62-68). Like all animal models for biomedical research, there are limitations to their use in HIV research. Some of these limitations are intrinsic and are related to the size and biology of the animal, including the relatively small volume of blood plasma that can be obtained for viral load analysis, the limited amount of peripheral blood cells that can be used for in vitro functional analysis, and the relatively short lifespan of the animal. In this regard, humanized mice can be considered a useful accelerated model for the rapid evaluation of relevant interventions. Other limitations are related to the nature of the xenografts between humans and mice. For example, the structure of secondary lymphoid tissues found in humanized mice does not fully replicate the structures observed in human tissues. Moreover, some highly engrafted animals develop a wasting disease $(47,69)$. However, these problems have been addressed in humanized mice generated in new immunodeficient mouse strains (55). Even though most humanized mouse models are capable of mounting adaptive immune responses, these are not optimal, and efforts are underway to improve them $(46,47,70,71)$. Nevertheless, the current humanized mouse models have been used extensively to test the efficacy of numerous immune approaches to control HIV replication and kill infected cells in vivo $(67,72-74)$. 


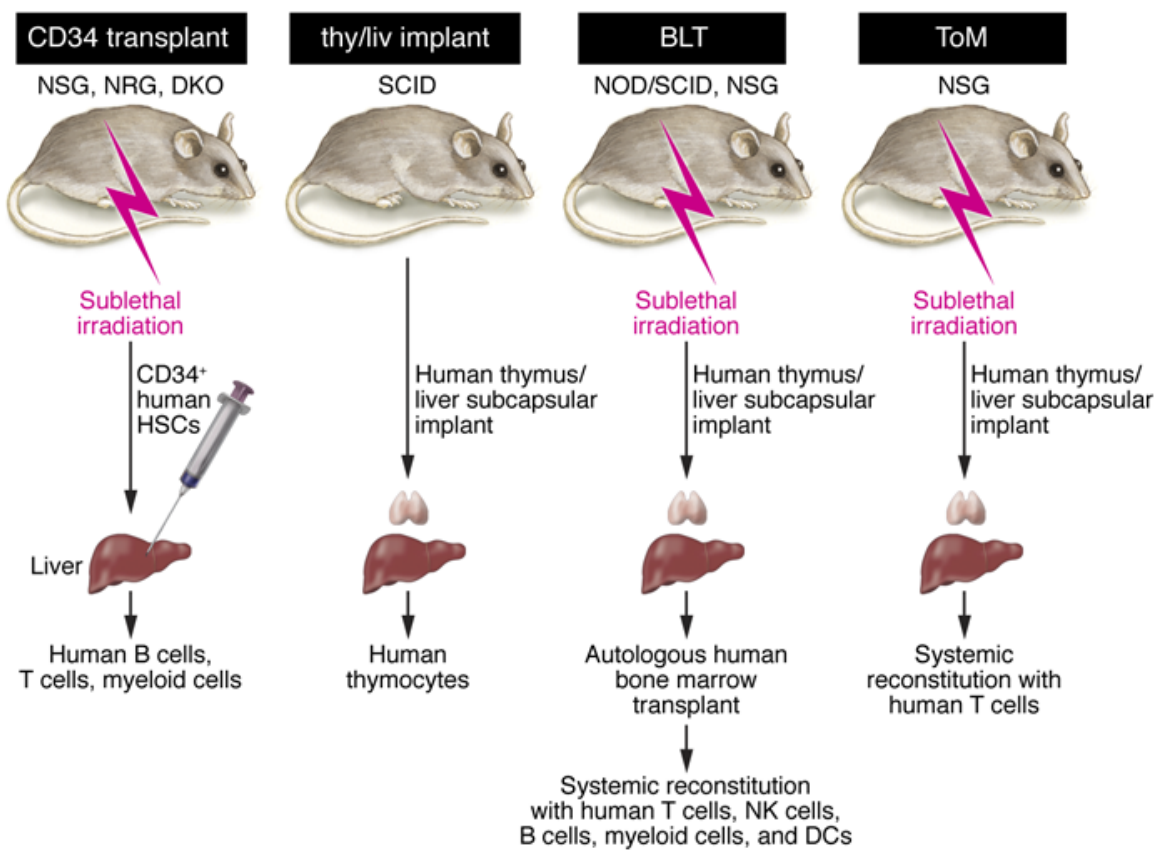

Figure 1. Different humanized mouse models used for in vivo analysis of HIV latency and persistence. The different strains of animals commonly used to generate humanized mice after $\mathrm{CD}_{3} 4^{+}$cell transplantation and/or tissue implantation are indicated. In all cases, mice are preconditioned with radiation prior to transplantation with $\mathrm{CD}_{3} 4^{+}$cells and tissue implantation. Note that in some instances, such as in the case of BLT mice and ToM, the same strain of mouse is reconstituted with different sets of human hematopoietic cells.

\section{Analysis of HIV persistence in SCID-hu thy/liv humanized mice}

SCID-hu thy/liv mice were originally described by McCunne et al. as a murine model for the analysis of human hematolymphoid differentiation and function (75). Shortly thereafter, Namikawa et al. demonstrated that these mice were susceptible to HIV infection when virus was directly injected into the human thymic organoid that develops in these animals (76). The utility of this model to investigate the genesis of HIV latency was demonstrated by Brooks et al. (77), who used SCID-hu thy/liv mice to show that HIV latency could be generated during thymopoiesis and that this process could contribute to the systemic establishment of HIV latency in peripheral organs. The authors postulated that $\mathrm{CD} 4$ and $\mathrm{CD} 8$ double-positive thymocytes were targets of HIV infection and that the transcriptional silencing that occurs during thymopoiesis silenced the HIV promoter, resulting in latently infected, single-positive CD4 T cells capable of exiting the thymus into the periphery and seeding the spleen. Consistent with their hypothesis, the authors were also able to detect HIV in CD8 single-positive cells. Ex vivo induction of both cell types with either anti-human CD3 Abs or cytokines resulted in the production of virus. The authors concluded that HIV latency was readily established in thymocytes and that this process was a significant contributor to thymic and systemic HIV persistence. In subsequent work, the authors also investigated the role of NF- $\kappa \mathrm{B}$ in HIV reactivation and used in vivo-generated, latently infected cells for ex vivo activation studies aimed at investigating $\mathrm{T}$ cell-signaling pathways that stimulated latent HIV in primary cells (78). Further analysis demonstrated that latent HIV could be reactivated ex vivo using prostratin and $\operatorname{IL}-7(79,80)$. The SCID-hu thy/liv model was also used to demonstrate the efficacy of immunotoxins in killing latently infected cells after reactivation ex vivo (81) and in killing infected thymocytes in animals undergoing combination ART (82).

\section{Analysis of HIV latency and persistence in ToM} Despite its utility in the investigation of HIV infection and persistence in thymocytes, the SCID-hu thy/liv model is somewhat limited, given that SCID-hu thy/liv mice possess very few systemic human $\mathrm{T}$ cells and infection of the thymic organoid results in low levels of plasma viremia. The advent of a new and improved immunodeficient mouse strain has made it possible to expand the usefulness of this type of tissue implant model by providing increased levels of peripheral and systemic reconstitution with human $\mathrm{T}$ cells. Implantation of thy/liv tissue into NSG mice results in the development of a thymic organoid similar to the one that develops in SCID-hu thy/liv mice (83). However, in striking contrast with the virtual absence of human $\mathrm{T}$ cells in the periphery and tissues of SCID-hu thy/liv mice, NSG thy/liv mice have significant levels of human $\mathrm{T}$ cells in all tissues analyzed, including peripheral blood, spleen, thymus, lymph nodes, bone marrow, liver, and lung (83). What sets this model apart from the NSG mice transplanted with human CD34 ${ }^{+}$cells (described below) is the complete absence of human antigenpresenting cells (APCs). Specifically, NSG thy/liv mice are devoid of any human B cells, monocytes, macrophages, or DCs. Thus, these animals have been functionally designated as ToM (83). Unlike other humanized mouse models with high levels of systemic reconstitution with human T cells, ToM do not develop signs of graft-versus-host disease (GVHD) (83). ToM are susceptible to HIV infection and support high levels of viral replication, as determined by the analysis of plasma vRNA, and viremia is maintained for the lifespan of the animals. Consistent with the systemic distribution of human $\mathrm{CD}^{+} \mathrm{T}$ cells, HIV-infected cells can be found in all tissues examined. ART efficiently suppresses viremia in these mice, and structured treatment interruption results in viral rebound (83). In order to determine whether HIV latency is established in this model, cells obtained from suppressed animals were harvested from multiple tissues, 
and resting $\mathrm{CD}^{+} \mathrm{T}$ cells were isolated for ex vivo induction assays. Using the same methodology previously described for the quantitation of HIV latency in humans (84-86), ToM were shown to establish HIV latency with a frequency similar to that observed in chronically infected patients receiving ART (83).

\section{In vivo evaluation of broadly neutralizing Abs for HIV treatment}

Unlike most conventional drugs used for the treatment of HIV/ AIDS, Abs have numerous functions that, in addition to neutralizing infectious virus, can engage multiple aspects of the immune system to control infection and possibly kill infected cells in vivo (87-89). Some recently discovered Abs have extremely broad in vitro neutralization activity. When evaluated individually for their ability to control infection in vivo in humanized mice, these Abs were shown to provide a short (6-7 days) and variable $\left(0.2-1.1 \log _{10}\right)$ reduction in peripheral blood plasma viral load levels (73). Viral replication in the presence of sustained Ab levels was associated with mutations that were mapped to the respective Ab-binding site (73). Similar results were obtained when infected mice were treated with a combination of three Abs, except that there was partial control of infection in 3 of the 12 treated animals. When a combination of five mAbs was administered to infected mice, a dramatic drop in viral load to below the level of detection (800 copies of vRNA per milliliter of plasma) was noted in 14 of 14 treated animals. This viral suppression was maintained for up to 60 days. Despite strong control of viremia in these mice by the $\mathrm{Ab}$ combination, viral rebound occurred an average of 60 days after therapy interruption. These results demonstrate the utility of humanized mice for the in vivo evaluation of broadly neutralizing Abs to suppress viremia. A second combination of three Abs in this model exhibited an even greater reduction in viral load, which was maintained for the entire course of treatment and accompanied by a reduction $\left(0.8 \log _{10}\right)$ in vDNA levels in peripheral blood (67). Continuous administration of this $\mathrm{Ab}$ combination to animals previously treated with ART prevented viral rebound in some animals after ART cessation. As serum Ab levels decreased, the authors observed viral rebound in all animals. Given these highly encouraging results, single-Ab administration during and after ART was evaluated. In this case, selected Abs were able to control viremia in some, but not all, mice (67).

As demonstrated by the studies described above, intermittent dosing of Abs is associated with systemic Ab levels that vary between animals. Alternative approaches for the delivery of Abs have been evaluated in humanized animal models for HIV prevention (90-92). The most common way to deliver sustained levels of Abs has been the use of adeno-associated virus (AAV) vectors. With regard to the use of sustained delivery of Abs for HIV cure approaches, it is important to mention that administration of single Abs via AAV transduction in vivo was shown to result in better suppression in reconstituted NRG mice than intermittent dosing (67).

\section{Evaluation of strategies to induce the latent HIV reservoir}

Latent reservoirs of HIV are long lived and not directly affected by current ARTs $(15,93,94)$. Given that latently infected cells are presumed to be transcriptionally silent, they are also presumed to not express HIV antigens and are therefore not expected to be recognized by the immune system, therapeutic Abs, or ex vivogenerated chimeric antigen receptor (CAR) T cells. In order to engage any of these approaches in the destruction of infected cells, latently infected cells must first be induced to express HIV, a process known as latency reversal. Three latency-reversing agents (LRAs), vorinostat, I-BET151, and cytotoxic T lymphocyte antigen 4 (CTLA-4), were evaluated in humanized NRG mice for their ability to prevent or delay viral rebound (72). LRAs were administered individually or in combination to infected NRG humanized mice that were previously suppressed for 20 days with a combination of three mAbs (3BNC117, 10-1074, and PG16) (72). LRAs were administered for 5 to 14 days, and the mice were monitored for viral rebound for an additional 45 to 85 days. Individual inducers had no discernible effect on viral rebound: of the suppressed infected animals receiving a single inducer, 31 of 33 mice rebounded. In contrast, of the animals receiving the combination of three different inducers that were under evaluation for an additional 62 to 105 days, $57 \%$ failed to show viral rebound, suggesting a possible reduction in the viral reservoir (72).

\section{Analysis of HIV latency in DKO mice reconstituted with human $\mathrm{CD}_{3} 4^{+}$cells}

DKO humanized mice efficiently replicate HIV after an i.v. or vaginal exposure $(66,95-98)$. Using a detailed pharmacokinetic analysis of plasma drug levels, Chaudhary et al. implemented a drug regimen consisting of tenofovir, emtricitabine, and the strand-transfer inhibitor L-870812, which was capable of efficiently suppressing HIV replication in most HIV-infected DKO humanized mice, as determined by plasma viral load analysis (99). In animals in which viral suppression was not complete, the authors were able to demonstrate the development of drug-resistant viruses. Discontinuation of therapy after viral suppression resulted in rapid viral rebound and loss of peripheral CD4 ${ }^{+}$ $\mathrm{T}$ cells, demonstrating that this model recapitulated key aspects of human HIV infection. In a subsequent communication that used the same methodology, Choudhary et al. investigated the establishment of HIV latency in infected DKO humanized mice (100). In these studies, the investigators isolated resting $\mathrm{CD} 4^{+}$ $\mathrm{T}$ cells from the tissues of untreated and ART-suppressed HIV-infected mice. The cells were incubated ex vivo with human IL-2 (hIL-2), or a combination of hIL-2 and phytohemagglutinin (PHA) to induce virus production. The virus was allowed to spread to allogeneic, CD8-depleted human peripheral blood mononuclear cells (PBMCs), as is routinely done to quantitate the levels of latently infected cells in HIV-infected patients undergoing suppressive ART $(85,86)$. These analyses indicated that, in this humanized mouse model, HIV latency was established and that the number of latently infected cells varied between 2 and 12 infectious units per million (IUPM) of resting $\mathrm{CD} 4^{+} \mathrm{T}$ cells, with a median of 8 IUPM. The authors also noted that, in some instances, no replication-competent virus could be induced in the ex vivo cultures. The failure to detect latent virus in these cultures was attributed to the small numbers of cells obtained from many of these animals. 


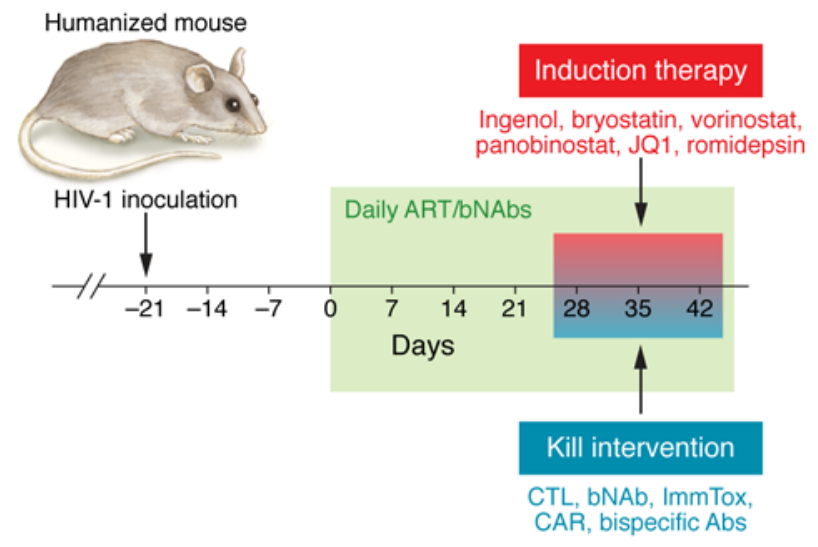

\section{Analysis of HIV latency and persistence in BLT mice}

BLT humanized mice are bioengineered by sandwiching a piece of human liver tissue between two pieces of human thymus tissue and implanting it under the kidney capsule of preconditioned immunodeficient mice. The mice then undergo bone marrow transplantation with autologous HSCs derived from the same liver tissue used for the implant (53). As described for SCID-hu thy/liv mice and for ToM, the thy/liv implant in BLT mice develops into a bona fide human thymic organoid into which human $\mathrm{T}$ cell progenitors produced in the bone marrow can migrate and differentiate through all stages of thymocyte development on human thymic epithelium in the context of HLA I and II (53). Bone marrow engraftment also results in the production of virtually all other human hematopoietic cell types, including those directly relevant to HIV research such as monocytes, macrophages, and DCs. Human cells in BLT mice are systemically distributed throughout all organs analyzed, including bone marrow, lymph nodes, spleen, thymus, liver, lung, female and male reproductive tracts, and the upper and lower digestive tracts (53, 61, 101-103). The wide distribution of human HIV target cells in mucosal sites renders BLT mice susceptible to rectal, vaginal, and oral HIV infection $(55,61-63,65,103,104)$. Mucosal or parenteral exposure of BLT mice to HIV results in systemic infection and the production of both human $\mathrm{Ab}$ and $\mathrm{T}$ cell responses $(55,59-61)$.

Detailed pharmacokinetic analysis of plasma drug levels in BLT mice $(64,74)$ has resulted in ART drug combinations consisting of tenofovir, emtricitabine, and raltegravir that efficiently suppress virus in plasma to below-detection levels. Suppression is maintained as long as ART is administered; however, as discussed above for ToM, therapy interruption results in rapid viral rebound to levels similar to those present prior to therapy initiation (64). The ability to efficiently suppress viral replication in BLT mice has facilitated the ex vivo analysis of HIV latency and persistence. Using the methodology described above for similar experiments in HIV-infected DKO humanized mice (100), analysis of suppressed BLT mice demonstrates the presence of latently infected cells at a frequency of approximately 8 IUPM resting $\mathrm{CD} 4^{+} \mathrm{T}$ cells (64).

Longitudinal analysis of cell-associated vRNA levels in the tissues of BLT mice during ART treatment demonstrated a rapid initial decrease that plateaued approximately 28 days after ART initiation in all tissues analyzed (74). No further decrease in cell-associated vRNA levels was observed with continued treatment. Administration of an
Figure 2. Analysis of "kick and kill" strategies in humanized mice. Diagram illustrating the different steps in the process of evaluating induction and/or HIV-infected cell-killing strategies in humanized mice. Humanized mice are first exposed to HIV via parenteral or mucosal routes. Once systemic viremia has been established, antiretroviral drugs are administered either orally or via i.p. injection. Once HIV is suppressed below the limit of detection, mice can be treated with induction and/or killing approaches. Several candidates for induction and HIV-infected cell killing are shown. bNAbs, broadly neutralizing Abs; ImmTox, immunotoxins.

immunotoxin (a toxin conjugated with an Ab targeting the HIV Env protein) to ART-suppressed animals resulted in additional dramatic decreases in vRNA levels in tissues (74). Analysis of the numbers of RNA-producing cells in tissues demonstrated efficient in vivo killing of infected cells by immunotoxin treatment (74). Because this approach only targets the "active" viral reservoir (i.e., cells producing the Env antigen) and not latently infected cells, the effect of the immunotoxin on the latent reservoir was not analyzed. In summary, the BLT humanized mouse model has been shown to serve as an excellent platform for the evaluation of novel approaches to the elimination of HIV-infected cells in vivo.

\section{Summary and future directions}

The utility of humanized mice for the in vivo analysis of HIV persistence has been partially demonstrated. Humanized mice provide excellent models for the analysis of virtually all aspects of HIV persistence and eradication. These include (a) the evaluation of different types of highly relevant viruses for infection; (b) the analysis of latency and persistence by virtually all human cell types that are targets of HIV infection in both the periphery and tissues; (c) the evaluation of new and established antiretroviral drug interventions; and (d) the evaluation of novel induction and killing approaches (Figure 2). In addition, because humanized mice represent a complex system featuring virtually all cell types that are important for adaptive immune responses, they can be useful for the in vivo evaluation of novel approaches to curing HIV that are based on biological molecules as well as gene and cell therapies (105-108). Even though most of the humanized mouse models available to date are limited in their ability to mount effective $\mathrm{Ab}$ responses $(70,109)$, as indicated above, these mice have been shown to be very useful for evaluating the efficacy of HIV-specific Ab therapy and other immunotherapeutic interventions such as checkpoint inhibitors, cytokines, and so forth. (105, 110). The primary limitations of humanized mice in the study of HIV latency, persistence, and eradication are the short lifespan of these animals and the relatively low volumes of blood and other samples that can be obtained. In addition, it should always be kept in mind that the human HIV-infected cells are being studied in the context of a chimeric mouse/human xenograft; therefore, not all of the cellular interactions that occur in humans are fully recapitulated in these models. Furthermore, the interactions between the murine and human cells (that obviously are not occurring in 
humans) should be taken into consideration. Nevertheless, new and improved strains of mice and a better understanding of the interplay that occurs at the interface between the human immune system and that of the mouse will result in improvements that will continue to benefit the field of HIV cure research. At present, humanized mouse models can be considered excellent platforms for the in vivo evaluation of HIV eradication strategies.

\section{Acknowledgments}

I thank A. Wahl, P. Tsai, C. Lopez, T. Morgan, J. Xiong, and C. De for their comments and suggestions. This work was supported in part by
NIH grants AI33331, AI73146, AI96138, AI111899, MH108179, and AI96113 and by the UNC Center for AIDS Research (P30AI50410). I would like to acknowledge the editorial assistance of David Carroll of the North Carolina Translational and Clinical Sciences Institute (NC TraCS), through NIH grant award UL1TR001111.

Address correspondence to: J. Victor Garcia, Division of Infectious Diseases, UNC Center for AIDS Research, University of North Carolina School of Medicine, Genetic Medicine Building, Rm. 2043, Chapel Hill, North Carolina 27599-7042, USA. Phone: 919.843.9600; E-mail: Victor_garcia@med.unc.edu.
1. Chun TW, et al. Quantification of latent tissue reservoirs and total body viral load in HIV-1 infection. Nature. 1997;387(6629):183-188.

2. Chun TW, Finzi D, Margolick J, Chadwick $\mathrm{K}$, Schwartz D, Siliciano RF. In vivo fate of HIV-1-infected T cells: quantitative analysis of the transition to stable latency. Nat Med. 1995;1(12):1284-1290.

3. Finzi D, et al. Identification of a reservoir for HIV-1 in patients on highly active antiretroviral therapy. Science. 1997;278(5341):1295-1300.

4. Chun TW, Davey RT Jr, Engel D, Lane HC, Fauci AS. Re-emergence of HIV after stopping therapy. Nature. 1999;401(6756):874-875.

5 . Chun TW, et al. Presence of an inducible HIV-1 latent reservoir during highly active antiretroviral therapy. Proc Natl Acad Sci US A. 1997;94(24):13193-13197.

6. Davey RT Jr, et al. HIV-1 and T cell dynamics after interruption of highly active antiretroviral therapy (HAART) in patients with a history of sustained viral suppression. Proc Natl Acad Sci U S A. 1999;96(26):15109-15114.

7. Le Douce V, Herbein G, Rohr O, Schwartz C. Molecular mechanisms of HIV-1 persistence in the monocyte-macrophage lineage. Retrovirology. 2010;7:32.

8. Sonza S, et al. Monocytes harbour replication-competent, non-latent HIV-1 in patients on highly active antiretroviral therapy. AIDS. 2001;15(1):17-22.

9. Eisele E, Siliciano RF. Redefining the viral reservoirs that prevent HIV-1 eradication. Immunity. 2012;37(3):377-388.

10. Laird GM, et al. Rapid quantification of the latent reservoir for HIV-1 using a viral outgrowth assay. PLoS Pathog. 2013;9(5):e1003398.

11. Archin NM, Sung JM, Garrido C, Soriano-Sarabia $\mathrm{N}$, Margolis DM. Eradicating HIV-1 infection: seeking to clear a persistent pathogen. Nat Rev Microbiol. 2014;12(11):750-764.

12. Finzi $\mathrm{D}$, et al. Latent infection of $\mathrm{CD} 4^{+} \mathrm{T}$ cells provides a mechanism for lifelong persistence of HIV-1, even in patients on effective combination therapy. Nat Med. 1999;5(5):512-517.

13. Siliciano JD, et al. Long-term follow-up studies confirm the stability of the latent reservoir for HIV-1 in resting $\mathrm{CD}^{+} \mathrm{T}$ cells. Nat Med. 2003;9(6):727-728.

14. Siliciano JD, Siliciano RF. Enhanced culture assay for detection and quantitation of latently infected, resting $\mathrm{CD}^{+}{ }^{+} \mathrm{T}$-cells carrying replication-competent virus in HIV-1-infected individuals. Methods
Mol Biol. 2005;304:3-15.

15. Blankson JN, et al. Biphasic decay of latently infected $\mathrm{CD}^{+} \mathrm{T}$ cells in acute human immunodeficiency virus type 1 infection. J Infect Dis. 2000;182(6):1636-1642.

16. Decloedt EH, Rosenkranz B, Maartens G, Joska J. Central nervous system penetration of antiretroviral drugs: pharmacokinetic, pharmacodynamic and pharmacogenomic considerations. Clin Pharmacokinet. 2015;54(6):581-598.

17. Calcagno A, Di Perri G, Bonora S. Pharmacokinetics and pharmacodynamics of antiretrovirals in the central nervous system. Clin Pharmacokinet. 2014;53(10):891-906

18. Tucker JD, Rennie S, Social, Ethical Working Group on HIVC. Social and ethical implications of HIV cure research. AIDS. 2014;28(9):1247-1250.

19. Eyal N, Kuritzkes DR. Challenges in clinical trial design for HIV-1 cure research. Lancet. 2013;382(9903):1464-1465.

20. Bibollet-Ruche F, et al. Efficient SIVcpz replication in human lymphoid tissue requires viral matrix protein adaptation. JClin Invest. 2012;122(5):1644-1652.

21. Watanabe M, et al. A chimpanzee-passaged human immunodeficiency virus isolate is cytopathic for chimpanzee cells but does not induce disease. J Virol. 1991;65(6):3344-3348.

22. Alter HJ, et al. Transmission of HTLV-III infection from human plasma to chimpanzees: an animal model for AIDS. Science. 1984;226(4674):549-552.

23. Gajdusek DC, et al. Infection of chimpanzees by human T-lymphotropic retroviruses in brain and other tissues from AIDS patients. Lancet. 1985;1(8419):55-56.

24. Fultz PN, et al. Persistent infection of chimpanzees with human T-lymphotropic virus type III/ lymphadenopathy-associated virus: a potential model for acquired immunodeficiency syndrome. J Virol. 1986;58(1):116-124.

25. Tervo HM, Keppler OT. High natural permissivity of primary rabbit cells for HIV-1, with a virion infectivity defect in macrophages as the final replication barrier. J Virol. 2010;84(23):12300-12314

26. Baumann JG, et al. Murine T cells potently restric human immunodeficiency virus infection. J Virol. 2004;78(22):12537-12547.

27. Ambrose Z, KewalRamani VN, Bieniasz PD, Hatziioannou T. HIV/AIDS: in search of an animal model. Trends Biotechnol. 2007;25(8):333-337.

28. Shibata R, Sakai H, Kawamura M, Tokunaga K, Adachi A. Early replication block of human immu- nodeficiency virus type 1 in monkey cells. J Gen Virol. 1995;76(pt 11):2723-2730.

29. Keppler OT, et al. Progress toward a human CD4/CCR5 transgenic rat model for de novo infection by human immunodeficiency virus type 1. JExp Med. 2002;195(6):719-736

30. Harris RS, Hultquist JF, Evans DT. The restriction factors of human immunodeficiency virus. J Biol Chem. 2012;287(49):40875-40883.

31. Malim MH, Bieniasz PD. HIV Restriction Factors and Mechanisms of Evasion. Cold Spring Harb Perspect Med. 2012;2(5):a006940.

32. Stremlau M, Owens CM, Perron MJ, Kiessling M, Autissier P, Sodroski J. The cytoplasmic body component TRIM5alpha restricts HIV-1 infection in Old World monkeys. Nature. 2004;427(6977):848-853.

33. Perez-Caballero D, Hatziioannou T, Yang A, Cowan S, Bieniasz PD. Human tripartite motif 5 alpha domains responsible for retrovirus restriction activity and specificity. J Virol. 2005;79(14):8969-8978.

34. Sawyer SL, Wu LI, Emerman M, Malik HS. Positive selection of primate TRIM5alpha identifies a critical species-specific retrovira restriction domain. Proc Natl Acad Sci US A. 2005;102(8):2832-2837.

35. Mariani R, et al. Species-specific exclusion of APOBEC3G from HIV-1 virions by Vif. Cell. 2003;114(1):21-31.

36. $\mathrm{Xu} \mathrm{H}$, et al. A single amino acid substitution in human APOBEC3G antiretroviral enzyme confers resistance to HIV-1 virion infectivity factor-induced depletion. Proc Natl Acad Sci U S A. 2004;101(15):5652-5657.

37. Dick JE. Immune-deficient mice as models of normal and leukemic human hematopoiesis. Cancer Cells. 1991;3(2):39-48.

38. Dick JE, Lapidot T, Pflumio F. Transplantation of normal and leukemic human bone marrow into immune-deficient mice: development of animal models for human hematopoiesis. Immunol Rev. 1991;124:25-43.

39. Hogan C, et al. Engraftment and development of human CD34(+)-enriched cells from umbilical cord blood in NOD/LtSz-scid/scid mice. Blood. 1997;90(1):85-96.

40. Vormoor J, et al. SCID mice as an in vivo model of human cord blood hematopoiesis. Blood Cells. 1994;20(2-3):316-320.

41. Wang JC, et al. High level engraftment of NOD/ SCID mice by primitive normal and leukemic hematopoietic cells from patients with chronic 
myeloid leukemia in chronic phase. Blood. 1998;91(7):2406-2414.

42. Kamel-Reid S, Dick JE. Engraftment of immune-deficient mice with human hematopoietic stem cells. Science. 1988;242(4886):1706-1709.

43. Denton PW, Garcia JV. Novel humanized murine models for HIV research. Curr HIV/AIDS Rep. 2009;6(1):13-19.

44. Brehm MA, Shultz LD, Greiner DL. Humanized mouse models to study human diseases. Curr Opin Endocrinol Diabetes Obes. 2010;17(2):120-125.

45. Denton PW, Garcia JV. Humanized Mouse Models of HIV Infection. AIDS Rev. 2011;13(3):135-148.

46. Shultz LD, Brehm MA, Garcia-Martinez JV, Greiner DL. Humanized mice for immune system investigation: progress, promise and challenges. Nat Rev Immunol. 2012;12(11):786-798.

47. Traggiai E, et al. Development of a human adaptive immune system in cord blood cell-transplanted mice. Science. 2004;304(5667):104-107.

48. Ishikawa F, et al. Development of functional human blood and immune systems in NOD/ SCID/IL2 receptor \{gamma\} chain(null) mice. Blood. 2005;106(5):1565-1573.

49. Shultz LD, et al. Human lymphoid and myeloid cel development in NOD/LtSz-scid IL2R $\gamma$ null mice engrafted with mobilized human hemopoietic stem cells. JImmunol. 2005;174(10):6477-6489.

50. Olesen R, Wahl A, Denton PW, Victor Garcia J. Immune reconstitution of the female reproductive tract of humanized BLT mice and their susceptibility to human immunodeficiency virus infection. J Reprod Immunol. 2011;88(2):195-203.

51. Wege AK, Melkus MW, Denton PW, Estes JD, Garcia JV. Functional and phenotypic characterization of the humanized BLT mouse model. Curr Top Microbiol Immunol. 2008;324:149-165.

52. Lan P, Tonomura N, Shimizu A, Wang S, Yang YG. Reconstitution of a functional human immune system in immunodeficient mice through combined human fetal thymus/liver and $\mathrm{CD} 34^{+}$cell transplantation. Blood. 2006;108(2):487-492.

53. Melkus MW, et al. Humanized mice mount specific adaptive and innate immune responses to EBV and TSST-1. Nat Med. 2006;12(11):1316-1322.

54. Pearson T, et al. Non-obese diabetic-recombination activating gene-1 (NOD-Rag1 null) interleukin (IL)-2 receptor common gamma chain (IL2r gamma null) null mice: a radioresistant model for human lymphohaematopoietic engraftment. Clin Exp Immunol. 2008;154(2):270-284.

55. Lavender KJ, et al. BLT-humanized C57BL/6 $\mathrm{Rag}^{-/ \gamma} \mathrm{C}^{-/-\mathrm{CD}} 47^{-/-}$mice are resistant to GVHD and develop B- and T-cell immunity to HIV infection. Blood. 2013;122(25):4013-4020.

56. Allam A, et al. TFH cells accumulate in mucosal tissues of humanized-DRAG mice and are highly permissive to HIV-1. Sci Rep. 2015;5:10443.

57. Danner R, et al. Expression of HLA class II molecules in humanized NOD.Rag1KO. IL2RgcKO mice is critical for development and function of human $\mathrm{T}$ and B cells. PLoS One. 2011;6(5):e19826.

58. Rongvaux A, et al. Human hemato-lymphoid system mice: current use and future potential for medicine. Аnпи Rev Immunol. 2013;31:635-674.

59. Brainard DM, et al. Induction of robust cellular and humoral virus-specific adaptive immune responses in human immunodeficiency virus-infected humanized BLT mice. J Virol. 2009;83(14):7305-7321.

60. Dudek TE, et al. Rapid evolution of HIV-1 to functional $\mathrm{CD}^{+} \mathrm{T}$ cell responses in humanized BLT mice. Sci Transl Med. 2012;4(143):143ra98.

61. Sun Z, et al. Intrarectal transmission, systemic infection, and $\mathrm{CD} 4^{+} \mathrm{T}$ cell depletion in humanized mice infected with HIV-1. J Exp Med. 2007;204(4):705-714.

62. Denton PW, et al. Antiretroviral pre-exposure prophylaxis prevents vaginal transmission of HIV-1 in humanized BLT mice. PLoS Med. 2008;5(1):e16.

63. Denton PW, et al. Systemic administration of antiretrovirals prior to exposure prevents rectal and intravenous HIV-1 transmission in humanized BLT mice. PLoS One. 2010;5(1):e8829.

64. Denton PW, et al. Generation of HIV latency in humanized BLT mice. JVirol. 2012;86(1):630-634

65. Denton PW, et al. One percent tenofovir applied topically to humanized BLT mice and used according to the CAPRISA 004 experimental design demonstrates partial protection from vaginal HIV infection, validating the BLT model for evaluation of new microbicide candidates. JVirol. 2011;85(15):7582-7593.

66. Nischang M, et al. Humanized mice recapitulate key features of HIV-1 infection: a novel concept using long-acting anti-retroviral drugs for treating HIV-1. PLoS One. 2012;7(6):e38853.

67. Horwitz JA, et al. HIV-1 suppression and durable control by combining single broadly neutralizing antibodies and antiretroviral drugs in humanized mice. Proc Natl Acad Sci U S A. 2013;110(41):16538-16543.

68. Kovarova M, et al. Nanoformulations of rilpivirine for topical pericoital and systemic coitus-independent administration efficiently prevent HIV transmission. PLoS Pathog. 2015;11(8):e1005075.

69. Greenblatt MB, Vbranac V, Tivey T, Tsang $\mathrm{K}$, Tager AM, Aliprantis AO. Graft versus host disease in the bone marrow, liver and thymus humanized mouse model. PLoS One. 2012;7(9):e44664.

70. Martinez-Torres F, Nochi T, Wahl A, Garcia JV, Denton PW. Hypogammaglobulinemia in BLT humanized mice - an animal model of primary antibody deficiency. PLoS One. 2014;9(10):e108663.

71. Karpel ME, Boutwell CL, Allen TM. BLT humanized mice as a small animal model of HIV infection. Curr Opin Virol. 2015;13:75-80.

72. Halper-Stromberg A, et al. Broadly neutralizing antibodies and viral inducers decrease rebound from HIV-1 latent reservoirs in humanized mice. Cell. 2014;158(5):989-999.

73. Klein F, et al. HIV therapy by a combination of broadly neutralizing antibodies in humanized mice. Nature. 2012;492(7427):118-122.

74. Denton PW, et al. Targeted cytotoxic therapy kills persisting HIV infected cells during ART. PLoS Pathog. 2014;10(1):e1003872.

75. McCune JM, Namikawa R, Kaneshima H, Shultz LD, Lieberman M, Weissman IL. The SCID-hu mouse: murine model for the analysis of human hematolymphoid differentiation and function. Science. 1988;241(4873):1632-1639.

76. Namikawa R, Kaneshima H, Lieberman $\mathrm{M}$, Weissman IL, McCune JM. Infection of the SCID-hu mouse by HIV-1. Science. 1988;242(4886):1684-1686.

77. Brooks DG, Kitchen SG, Kitchen CM, Scripture-Adams DD, Zack JA. Generation of HIV latency during thymopoiesis. Nat Med. 2001;7(4):459-464.

78. Brooks DG, Arlen PA, Gao L, Kitchen CM, Zack JA. Identification of $\mathrm{T}$ cell-signaling pathways that stimulate latent HIV in primary cells. Proc Natl Acad Sci U S A. 2003;100(22):12955-12960.

79. Scripture-Adams DD, Brooks DG, Korin YD, Zack JA. Interleukin-7 induces expression of latent human immunodeficiency virus type 1 with minimal effects on T-cell phenotype. J Virol. 2002;76(24):13077-13082.

80. Korin YD, Brooks DG, Brown S, Korotzer A, Zack JA. Effects of prostratin on T-cell activation and human immunodeficiency virus latency. J Virol. 2002;76(16):8118-8123.

81. Brooks DG, et al. Molecular characterization, reactivation, and depletion of latent HIV. Immunity. 2003;19(3):413-423.

82. Goldstein H, Pettoello-Mantovani M, Bera TK, Pastan IH, Berger EA. Chimeric toxins targeted to the human immunodeficiency virus type 1 envelope glycoprotein augment the in vivo activity of combination antiretroviral therapy in thy/liv-SCID-Hu mice. J Infect Dis. 2000;181(3):921-926.

83. Honeycutt JB, Wahl A, Archin N, Choudhary S, Margolis D, Garcia JV. HIV-1 infection, response to treatment and establishment of viral latency in a novel humanized $\mathrm{T}$ cell-only mouse (TOM) model. Retrovirology. 2013;10(1):121.

84. Archin NM, et al. Valproic acid without intensified antiviral therapy has limited impact on persistent HIV infection of resting $\mathrm{CD} 4^{+} \mathrm{T}$ cells. AIDS. 2008;22(10):1131-1135.

85. Archin NM, et al. Administration of vorinostat disrupts HIV-1 latency in patients on antiretroviral therapy. Nature. 2012;487(7408):482-485.

86. Lehrman G, et al. Depletion of latent HIV-1 infection in vivo: a proof-of-concept study. Lancet. 2005;366(9485):549-555.

87. Overbaugh J, Morris L. The Antibody Response against HIV-1. Cold Spring Harb Perspect Med. 2012;2(1):a007039.

88. Mascola JR, Montefiori DC. The role of antibodies in HIV vaccines. Annu Rev Immunol. 2010;28:413-444.

89. West AP Jr, Scharf L, Scheid JF, Klein F, Bjorkman PJ, Nussenzweig MC. Structural insights on the role of antibodies in HIV-1 vaccine and therapy. Cell. 2014;156(4):633-648.

90. Balazs AB, Chen J, Hong CM, Rao DS, Yang L, Baltimore D. Antibody-based protection against HIV infection by vectored immunoprophylaxis. Nature. 2012;481(7379):81-84.

91. Balazs AB, et al. Vectored immunoprophylaxis protects humanized mice from mucosal HIV transmission. Nat Med. 2014;20(3):296-300.

92. Balazs AB, West AP Jr. Antibody gene transfer for HIV immunoprophylaxis. Nat Immunol. 2013;14(1):1-5. 
93. Archin NM, et al. Immediate antiviral therapy appears to restrict resting $\mathrm{CD} 4^{+}$cell HIV- 1 infection without accelerating the decay of latent infection. Proc Natl Acad Sci U S A. 2012;109(24):9523-9528.

94. Chun TW, et al. HIV-infected individuals receiving effective antiviral therapy for extended periods of time continually replenish their viral reservoir. J Clin Invest. 2005;115(11):3250-3255.

95. Baenziger S, et al. Disseminated and sustained HIV infection in CD34+ cord blood cell-transplanted Rag2 ${ }^{-/} \mathrm{\gamma c}^{-/}$mice. Proc Natl Acad Sci U S A. 2006;103(43):15951-15956.

96. Akkina R, et al. Humanized Rag1 $1^{-/} \gamma \mathrm{c}^{-/-}$mice support multilineage hematopoiesis and are susceptible to HIV-1 infection via systemic and vaginal routes. PLoS One. 2011;6(6).

97. Berges BK, Akkina SR, Folkvord JM, Connick E, Akkina R. Mucosal transmission of R5 and $\mathrm{X} 4$ tropic HIV-1 via vaginal and rectal routes in humanized Rag2 ${ }^{-/ \gamma} \mathrm{c}^{-/-}(\mathrm{RAG}-\mathrm{hu})$ mice. Virology. 2008;373(2):342-351.

98. Berges BK, Akkina SR, Remling L, Akkina R. Humanized Rag2 $\left(^{(-)}\right) \gamma \mathrm{c}^{(-/)}$(RAG-hu) mice can sustain long-term chronic HIV-1 infection lasting more than a year. Virology. 2009;397(1):100-103. 99. Choudhary SK, et al. Suppression of human immunodeficiency virus type 1 (HIV-1) viremia with reverse transcriptase and integrase inhibitors, $\mathrm{CD} 4^{+} \mathrm{T}$-cell recovery, and viral rebound upon interruption of therapy in a new model for HIV treatment in the humanized Rag2 $2^{-/}$\{gamma $\mathrm{g} \mathrm{c}^{-/}$ mouse. JVirol. 2009;83(16):8254-8258.

100.Choudhary SK, Archin NM, Cheema M, Dahl NP, Garcia JV, Margolis DM. Latent HIV-1 infection of resting CD4(+) T cells in the humanized Rag2 ${ }^{-/} \mathrm{\gamma c}^{-/}$ mouse. JVirol. 2012;86(1):114-120.

101.Denton PW, et al. IL-2 receptor $\gamma$-chain molecule is critical for intestinal T-cell reconstitution in humanized mice. Mucosal Immunol. 2012;5(5):555-566.

102. Nochi T, Denton PW, Wahl A, Garcia JV. Cryptopatches are essential for the development of human GALT. Cell Rep. 2013;3(6):1874-1884.

103. Wahl A, et al. Human breast milk and antiretrovirals dramatically reduce oral HIV-1 transmission in BLT humanized mice. PLoS Pathog. 2012;8(6):e1002732.

104. Chateau ML, Denton PW, Swanson MD, McGowan I, Garcia JV. Rectal transmission of transmitted/founder HIV-1 is efficiently prevented by topical $1 \%$ tenofovir in BLT humanized mice. PLoS One. 2013;8(3):e60024.

105. Palmer BE, et al. In vivo blockade of the PD-1 receptor suppresses HIV-1 viral loads and improves $\mathrm{CD} 4^{+} \mathrm{T}$ cell levels in humanized mice. JImmunol. 2013;190(1):211-219.

106.Kitchen SG, et al. In vivo suppression of HIV by antigen specific $\mathrm{T}$ cells derived from engineered hematopoietic stem cells. PLoS Pathog. 2012;8(4):e1002649.

107.Zhen A, et al. HIV-specific immunity derived from chimeric antigen receptor-engineered stem cells. Mol Ther. 2015;23(8):1358-1367.

108. Shimizu S, et al. RNAi-mediated CCR5 knockdown provides HIV-1 resistance to memory T cells in humanized BLT mice. Mol Ther Nucleic Acids. 2015;4:e227.

109. Akkina R. Humanized mice for studying human immune responses and generating human monoclonal antibodies. Microbiol Spectr. 2014;2(2).

110.Seay K, et al. In vivo activation of human NK cells by treatment with an interleukin-15 superagonist potently inhibits acute in vivo HIV-1 infection in humanized mice. JVirol. 2015;89(12):6264-6274. 